\title{
Research Paper \\ Comparing the Effect of Endurance Exercise and High-Intensity Interval Exercise on Plasma Levels of Chemerin and Insulin Resistance in Obese Male Rats
}

\author{
*Mohammad Reza Asad ${ }^{1}$, ${ }^{\text {, Shokoufeh Kheradmand }{ }^{2} \text {, }}$, Narges Kheradmand ${ }^{3}$ (C) \\ 1. Department of Physical Education and Sport Sciences, Payame Noor University of Karaj, Karaj, Iran. \\ 2. PhD student in Exercise Physiology, Faculty of Sport Sciences, University of Mazandaran, Babolsar, Iran. \\ 3. MSc in Physical Education and Sport Sciences, Payame Noor University, Garmsar, Iran.
}

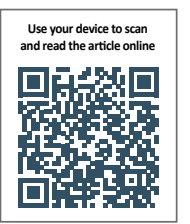

Cttation: Asad M, Kheradmand Sh, Kheradmand N. [Comparing the Effect of Endurance Exercise and High-Intensity Interval Exercise on Plasma Levels of Chemerin and Insulin Resistance in Obese Male Rats (Persian)]. Journal of Arak University of Medical Sciences(JAMS). 2019; 22(5):112-123. https://doi.org/10.32598/JAMS.22.5.112

doi https://doi.org/10.32598/JAMS.22.5.112

Key words:

Endurance exercise, high-intensity intermittent exercise, chemerin, insulin resistance, obese rats

\section{A B STRACT}

Background and Aim Exercise improves sensitivity to insulin by reducing some adipokines including chemerin. However, the effect of endurance exercise and high-intensity intermittent exercise on chemerin level and insulin resistance in obese male rats is still unknown.

Methods \& Materials In this study 27 male rats were randomly divided into 4 groups of sedentary control $(n=6)$, endurance exercise $(n=8)$, high-intensity intermittent exercise $(n=8)$ and untreated obese control $(n=5)$. Both endurance and high intensity intermittent exercise groups performed exercises for 8 weeks. The plasma level of chemerin was measured by ELISA method.

Ethical Considerations This study obtained its ethical approval form the Research Ethics Committee of Sport Sciences Research Institute (Code: IR.SSRI.REC.1398.55).

Results The chemerin level was significantly reduced in the endurance group compared to the untreated obese control group $(P<0.01)$, but it had no significant change compared to the sedentary control group. Moreover, the plasma level of chemerin in the high-intensity intermittent group was not significantly different from that of untreated obese control group and sedentary control group $(P>0.05)$ and chemerin level reduction in the endurance group was significant compared to the high-intensity intermittent group $(P<0.05)$. Furthermore, there was no significant difference in the insulin resistance reported in high-intensity intermittent group compared to that of endurance group ( $P>0.05)$, but its difference in high-intensity intermittent and endurance training groups was significant compared to that of untreated obese control and sedentary control groups $(P<0.05)$.

Conclusion Both endurance and high-intensity intermittent exercises can reduce insulin resistance which can be due to the reduction of chemerin plasma level and weight loss.

\footnotetext{
Extended Abstract

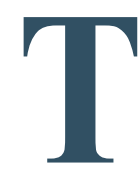

\section{Introduction}

he prevalence of obesity is associated with an increased risk of metabolic syndrome,

type 2 diabetes, and insulin resistance, so control and regulation of adipose tissue can prevent diseases associated with this tissue [1]. Adipose tissue plays an important role in energy balance management by secreting some adipokines including chemerin. Chemerin is secreted from visceral adipose tissue and liver [2] and plays an improtant

* Corresponding Author:

Mohammad Reza Asad, PhD.

Address: Department of Physical Education and Sport Sciences, Payame Noor University of Karaj, Karaj, Iran.

Tel: +98 (912) 4339086

E-mail: m_r_asad@yahoo.com
} 
role in macrophage recruitment to adipose tissue, development of inflammation and insulin resistance, leading to insulin stimulation and increased glucose uptake in adipocytes [3]. According to the observations, serum levels of chemerin are elevated in obese patients and are associated with various aspects of metabolic syndrome [4]. In addition, high concentrations of chemerin serum make it possible for pre-diabetic conditions which affects glucose homeostasis [5] and induce insulin resistance [7].

Regular exercise is a good strategy for treating many metabolic disorders, including insulin resistance and obesity, by improving insulin sensitivity via increasing the density and sensitivity of glucose transporter GLUT4 in skeletal muscle sarcoma [8] and affecting adipose tissue [9]. Twelve weeks of aerobic [4] and strength [3] exercises decreases insulin sensitivity in obese men by lowering chemerin serum level. There is also a reported significant decrease in chemerin plasma level after high-intensity intermittent training [15] and 12 weeks of aerobic interval training in 24 inactive students [6]; However, 8 weeks of sprint exercise have no significant effect on chemerin serum levels of female Sprague-Dawley rats [16]. Given that the effect of exercise on chemerin serum levels is somewhat contradictory [4], and the changes in its level are dependent on the type and intensity of the exercise, the present study aimed to investigate the effect of endurance exercise and high-intensity (HIIT) intermittent exercise on chemerin plasma level and insulin resistance in obese male rats.

\section{Materials and Methods}

Study samples were 27 male Wistar rats aged 35-45 days with a mean weight of $110 \pm 10 \mathrm{~g}$. They were randomly divided into 4 groups of sedentary control $(n=6)$, endurance exercise $(n=8)$, HIIT intermittent exercise $(n=8)$ and untreated obese control $(n=5)$. Both endurance and HIIT intermittent exercise groups performed exercises on a treadmill for 8 weeks. For the HIIT group, exercise protocol consisted of training sets with an intensity of $90 \% \mathrm{VO} 2$ max for $15-30$ seconds with a 1-min active resting interval. It reached from 5 sets in the first week to 12 sets in the last week. Total exercise activity was matched between the two training groups such that the intensity of exercise in the endurance group was $50-70 \% \mathrm{VO} 2 \mathrm{max}$. Accordingly, endurance group activity in the first week reached from 25 minutes at a speed of $15 \mathrm{~m} /$ min and with intensity of 50-70\% VO2max to $60 \mathrm{~min}$ at 22 $\mathrm{m} / \mathrm{min}$ speed. Forty-eight hours after the last training session, the rats of all three groups were anesthetized by intraperitoneal injection of ketamine and xylazine. Blood samples was collected from the heart of Rats directly. Plasma and insulin levels of chemerin were calculated by ELISA method, while plasma level of glucose was measured by enzymatic glucose oxidase, and insulin resistance index was calculated by HOMA-IR formula. After testing the normality of data distribution by Smirnov-Kolmogorov test and confirmation of the equality of variance assumption using Levene's test, ANOVA test was used for analyzing data and comparing groups. Scheffe's test was used for determining the location of differences between groups regarding chemerin level. For analyzing data related to insulin resistance, Welch's ANOVA test and Dant3 post hoc test were used.

\section{Results}

The chemerin level was significantly reduced in the endurance group compared to the untreated obese control group $(\mathrm{P}=0.006)$, but it had no significant change compared to the sedentary control group $(\mathrm{P}=0.590)$. Moreover, the plasma level of chemerin in the HIIT intermittent group was not significantly different from that of untreated obese control group $(\mathrm{P}=0.781)$ and sedentary control group $(\mathrm{P}=0.421)$ and chemerin level reduction in the endurance group was significant compared to the HIIT intermittent group $(\mathrm{P}=0.035)$. Furthermore, there was no significant difference in the insulin resistance reported in HIIT intermittent group compared to that of endurance group $(\mathrm{P}=0.825)$, but its difference in HIIT intermittent and endurance training groups was significant compared to that of untreated obese control and sedentary control groups $(\mathrm{P}<0.05)$.

\section{Conclusion}

Both endurance and HIIT intermittent exercises can decrease plasma levels of chemerin and insulin resistance. However, the reduction is partly dependent on the type of exercise, such that plasma levels of chemerin significantly decreased after a period of endurance exercise compared to HIIT intermittent exercise.

\section{Ethical Considerations}

\section{Compliance with ethical guidelines}

This study obtained its ethical approval form the Research Ethics Committee of Sport Sciences Research Institute (Code: IR.SSRI.REC.1398.55) and approved by Faculty of Physical Education and Sport Sciences, University of Tehran.

\section{Funding}

This study received no financial support from any organization. 
Authors' contributions

All authors contributed equally to the writing of this article.

Conflicts of interest

The authors declare no conflict of interest. 


\title{
مقايسه دو شيوه تمرين استقامتى و تناوبى شديد، در سطوح يالاسمايى كمرين و مقاومت به

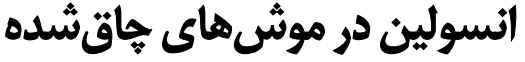

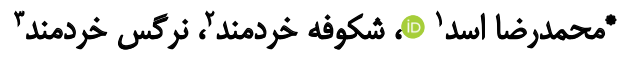 \\ 1. كروه تربيتبدنى و علوم ورزشى، دانشعاه بيام نور كرج، كرج، ايران.

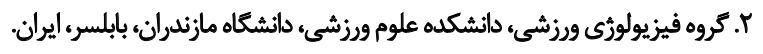

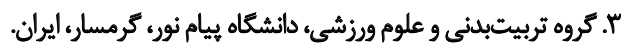

\begin{abstract}
سي

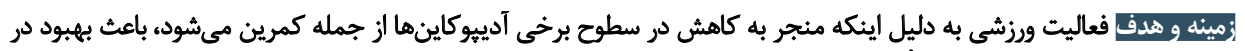

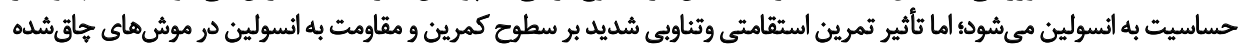
ناشناختهاست.

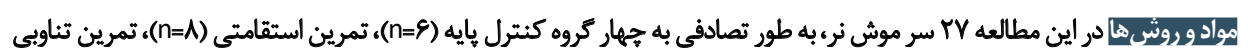

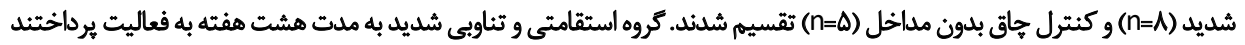

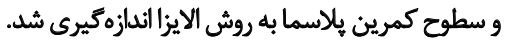

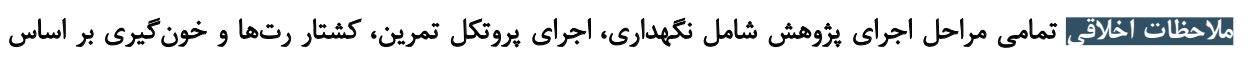

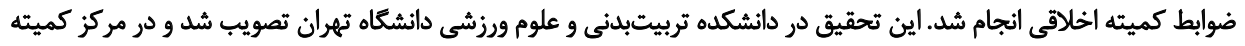

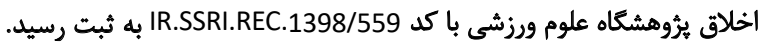

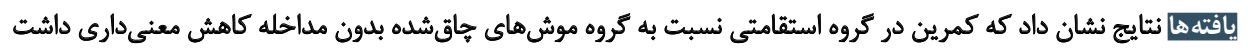

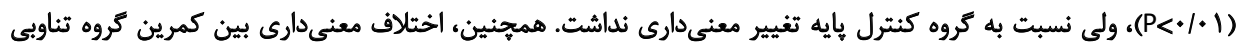

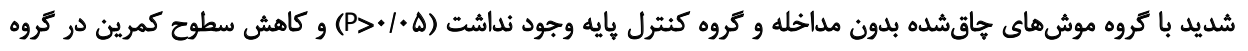

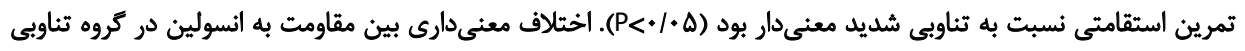

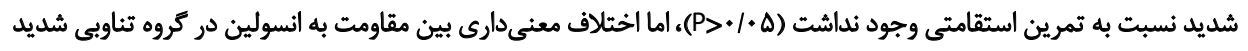

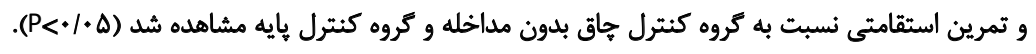

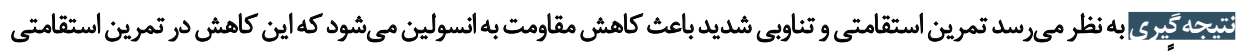
احتمالاً به واسطه كاهش وزن و كمرين سرمي اتفاتى مي افتئد.
\end{abstract}

اطلاعات مقاله:

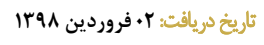

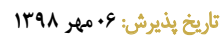

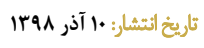

: تمرين استقامتي، ثمرين

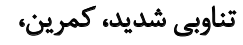
مقاومت به انسولين، موش هاى جاق

همهي در مديريت تعادل انرثي برعهله دارد. به عبارتى كمرين،

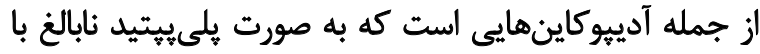

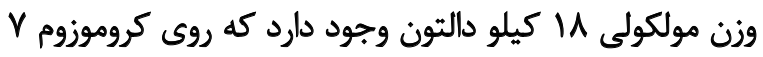

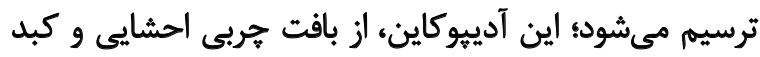

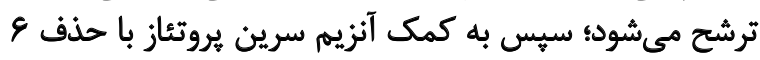

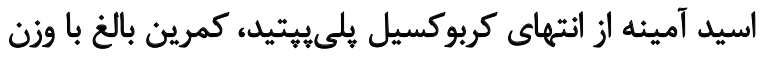

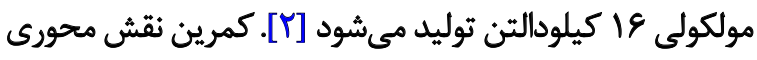

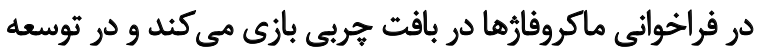

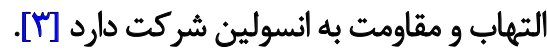
كمرين اثرات موضعى بر آديتوزنزيس دارد و منجر به تحريك

شيوع اخير جاقي، باعث نوعى نكراني عمده بهلداشتي انسانى

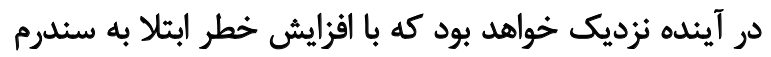

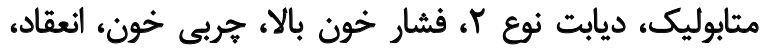
يروفايل سايتوكاينهاى التهابى و مقاومت به انسولين، همراه

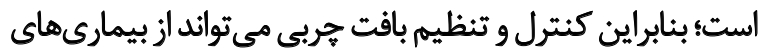

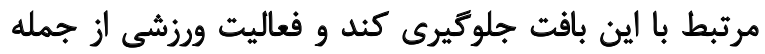

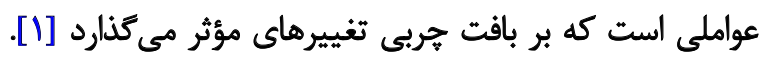

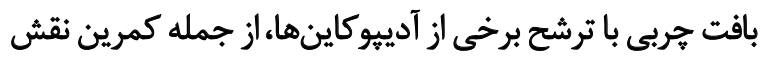

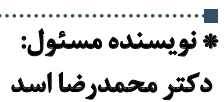

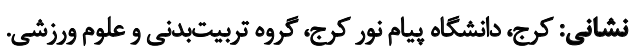

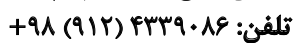
بست الكترونيكي: mer_asad@yahoo.com 
نشان مىدهد تمرينات ورزشى زير آستانه لاكتات سينتيك

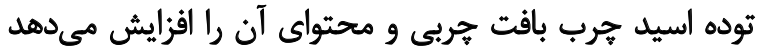

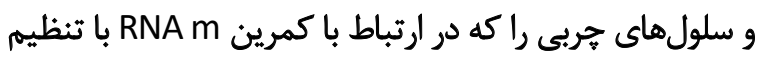

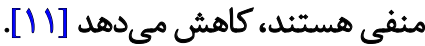

صارمى و همكاران، تأثير تمرينات قدرتى را بر سطوح كمرئ كمرين

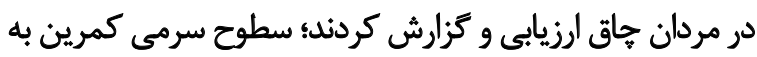

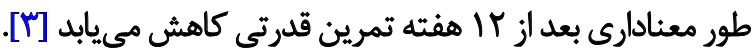

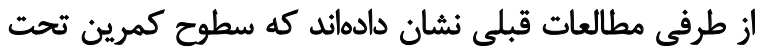

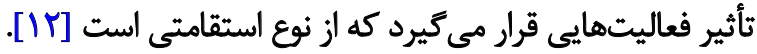

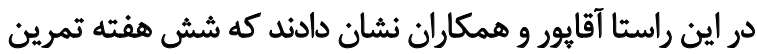

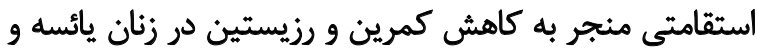

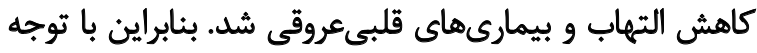

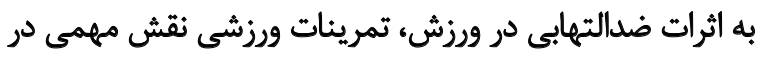

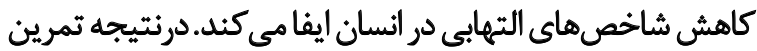

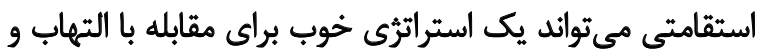

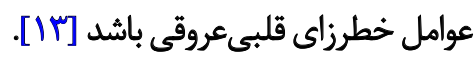

به طور كلى به نظر مىرسد مطالعات كذشته به طور عمدهاه،

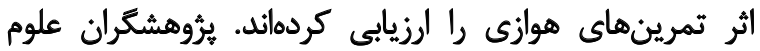

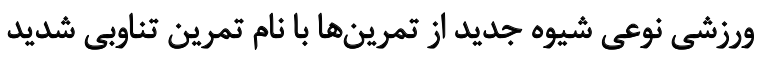

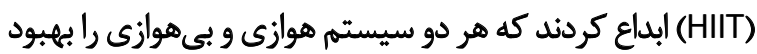

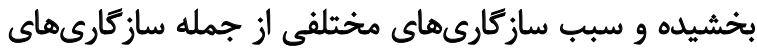

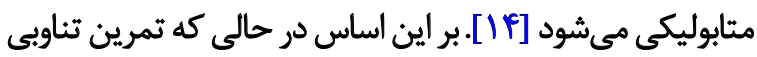

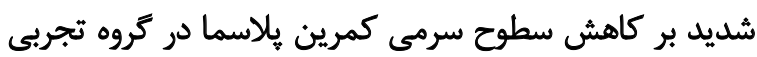

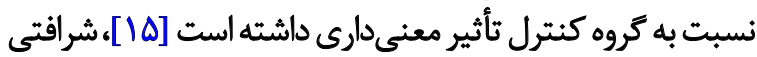

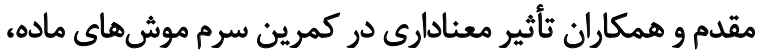

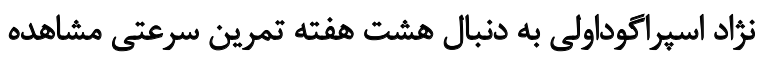

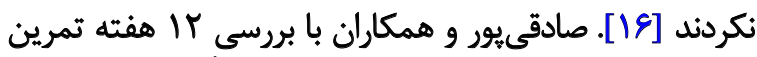

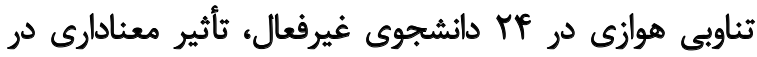

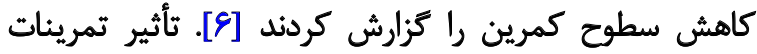

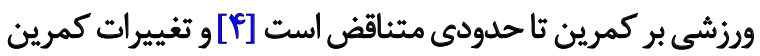

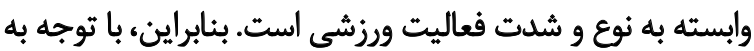

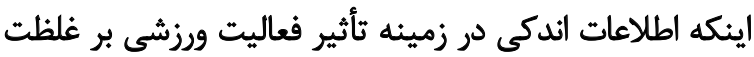

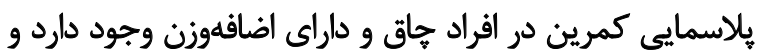

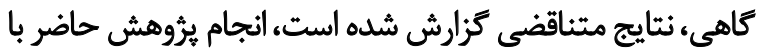

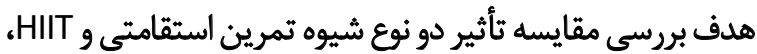

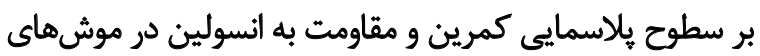

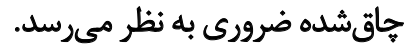

$$
\text { مواد و روشها }
$$

تحقيق حاضر از نظر روششناسى، تجربى و از نظر هدف،

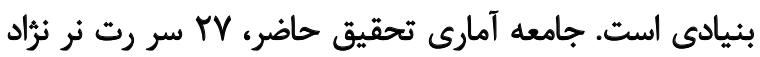

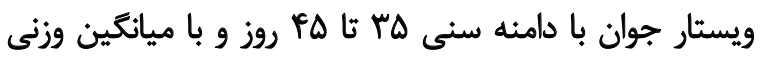

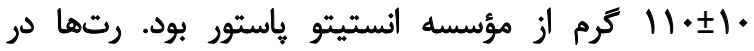

انسولين شده و جذب كلوكز در آديبوسيتها را افزيش ميدمهي

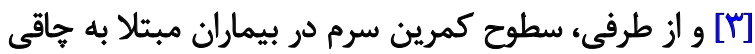

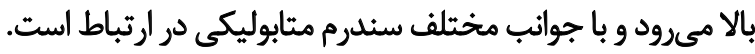

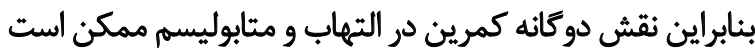

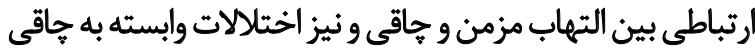

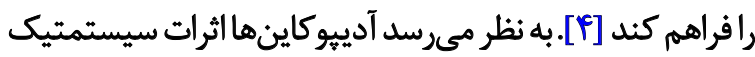

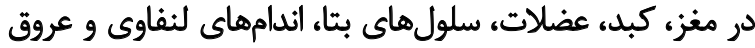

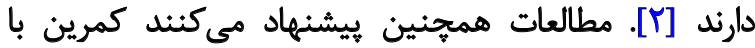

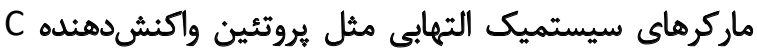

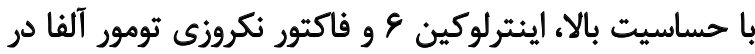

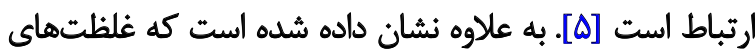

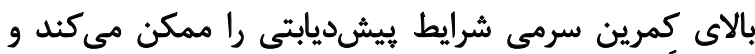

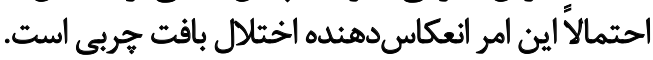

كرمجموع مطالعات انسانى نشان مى دهد كه كمرين بر هموستاز

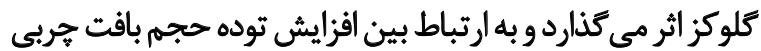

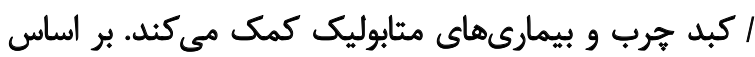

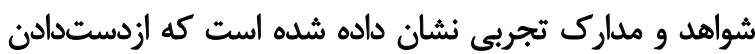

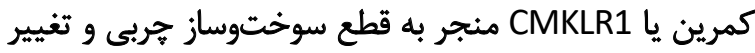

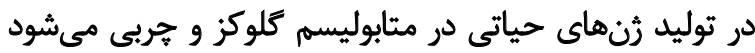

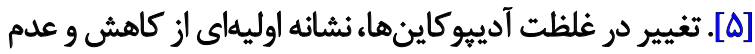

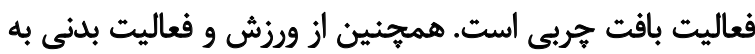

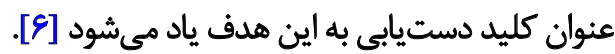

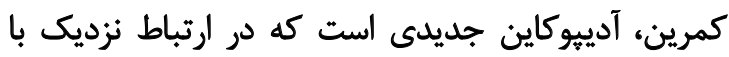

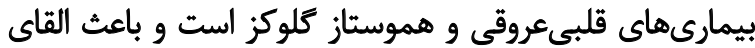

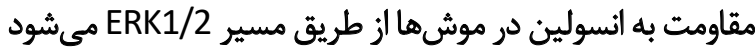

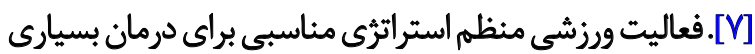

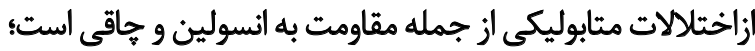

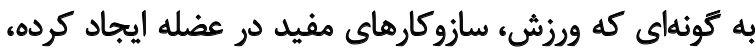

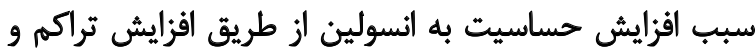

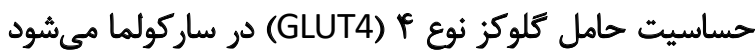

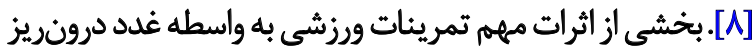

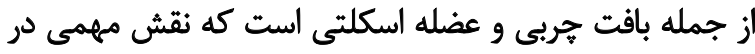

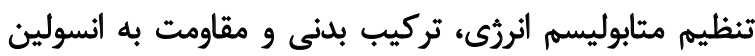

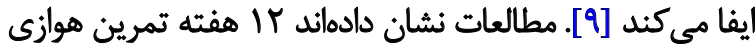
مىتواند از طريق كاهش سطوح كمرين سرم به بهبيود حساسيت

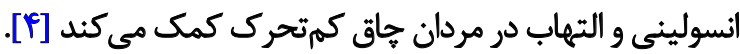

فعاليت بدنى و ورزش، اثر مطلوبى روى كاهش مقاومت بارئ بارئ

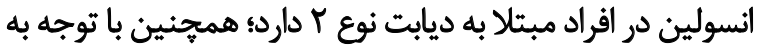

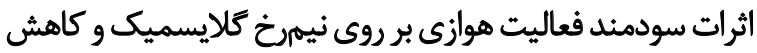

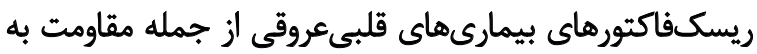

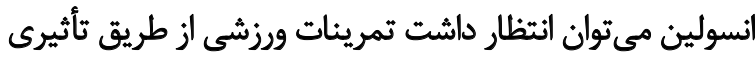

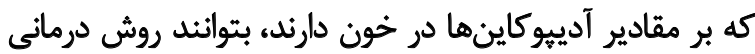

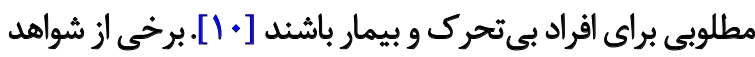


ادامه مراحل يُروهش به فريزر بادماي منهاي •Aد درجه سانتى كراد

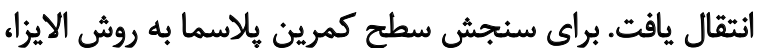

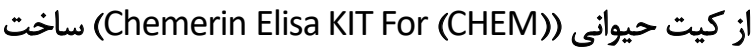
شركت آمريكاييجينى (USCN) كه از شركت تسنيمكستر

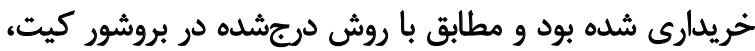

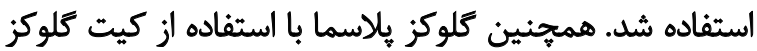

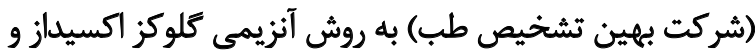

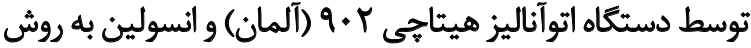

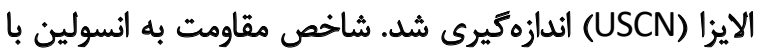

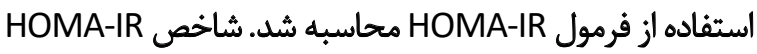

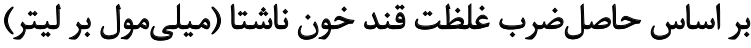

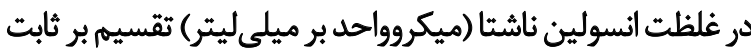

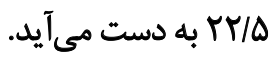

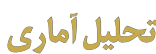

از آزمون كولموكروف|سميرنوف براى تعيين توزيع نرمال

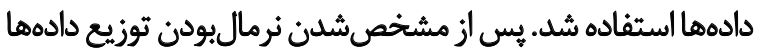

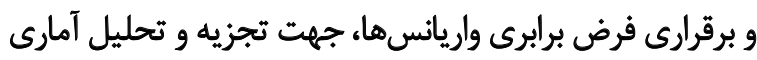

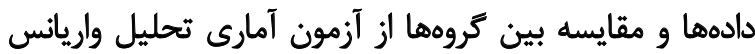

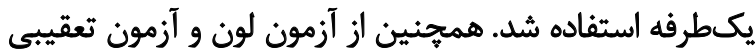

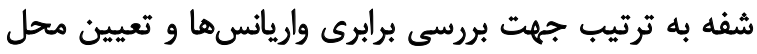

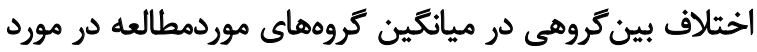

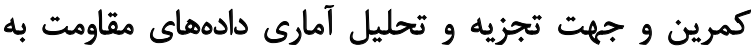

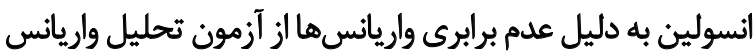
اصلاحشده' و آزمون تعقيبى Dant3 استفاده شد.

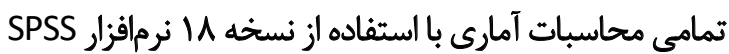

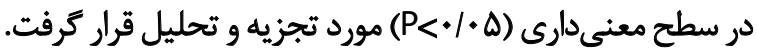

bedîl

تغييرات وزن بدن بين كروههاى تمرينى HIIT و استقامتى در مراحل مختلف يروهش در تصوير شماره إ آمده است.

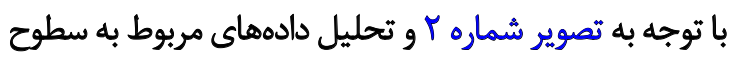

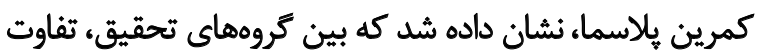

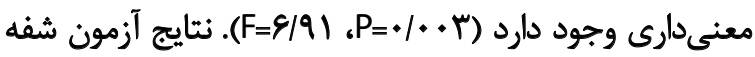
نشان داد كه سطوح كمرين در كروه تمرين استقامتى نسبت آندايت به گروه كنترل جاق (بدون مداخله

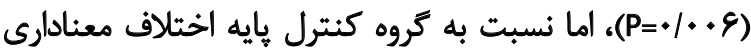

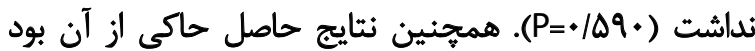

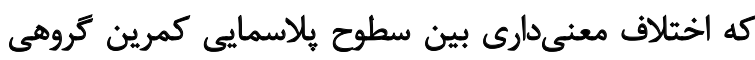

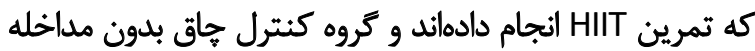

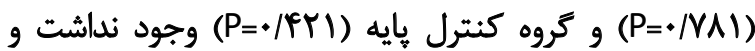

1. Welch
حيوان خانه دانشكده تربيت بدنى و علوم ورزشى دانشعاه تهران

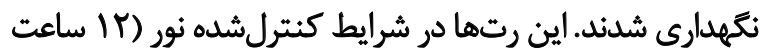

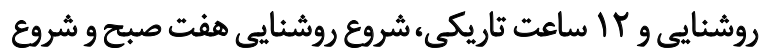

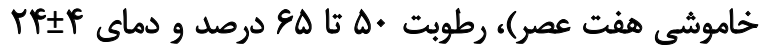

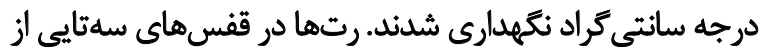

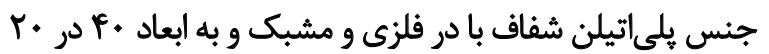

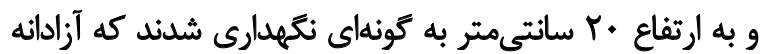

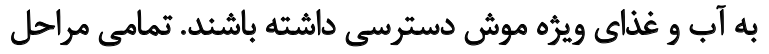

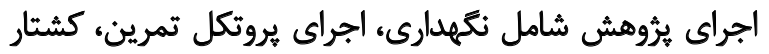
رتها و خون كيرى بر اساس ضوابط كميته اخلاقى انجام شد. در اين تحقيق، جهت اندازهيرى مقادير بايه از شش سر سرون

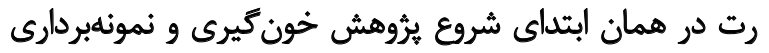

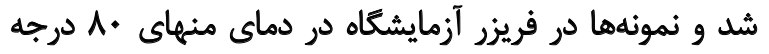

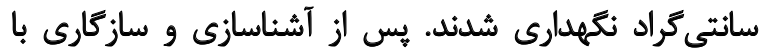

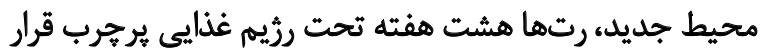

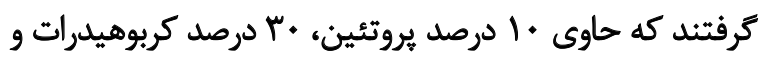

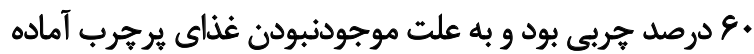

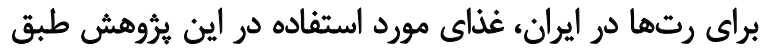

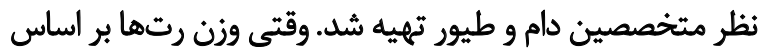

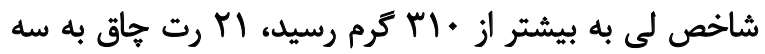

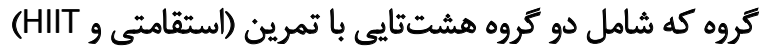

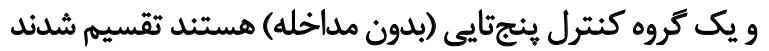
و تمرينات استقامتى و و HIIT آغاز شد.

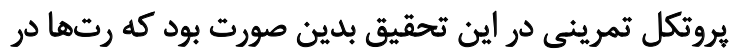

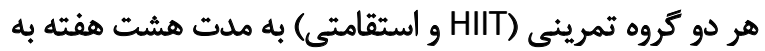

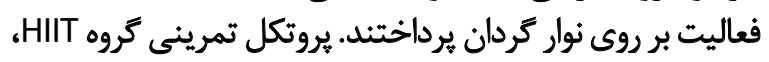

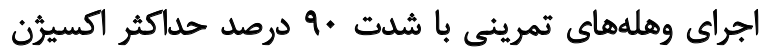

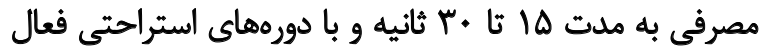

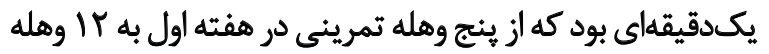

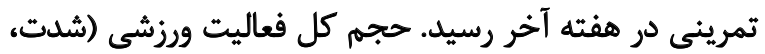

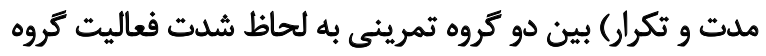

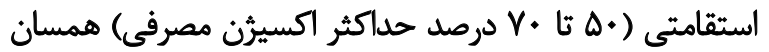

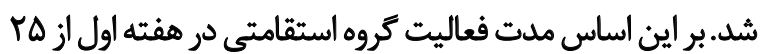

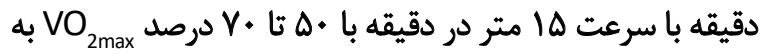

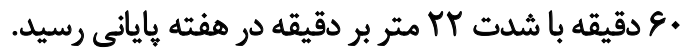
براى سنجش سطوح يلاسمايى كمرين و مقاومت به انسولين

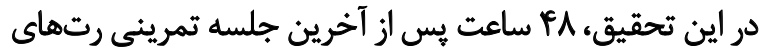

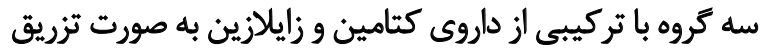

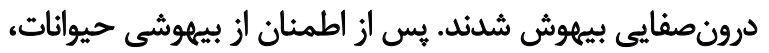

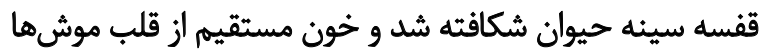

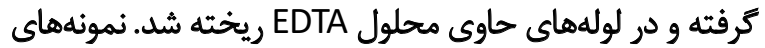

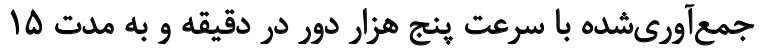

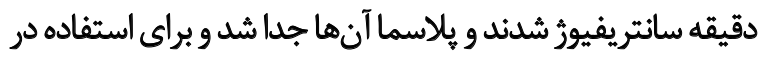




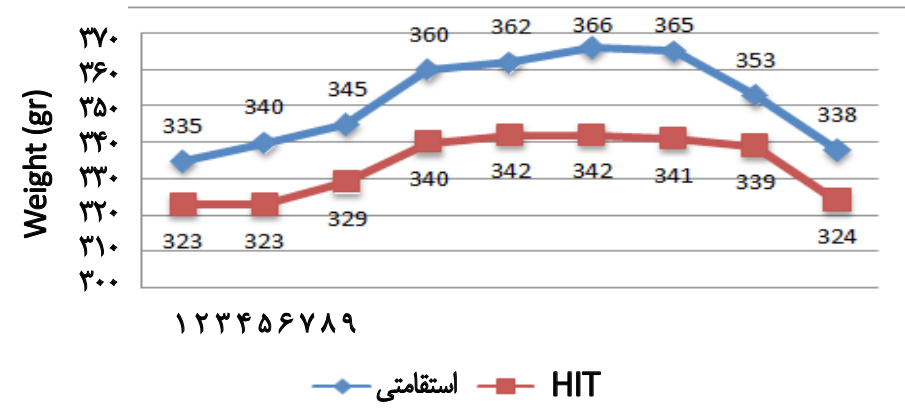

تصوير ا. تغييرات هفتكى وزن بدن در طول هشت هفته فعاليت ورزشى بين دو كروه استقامتى و HIIT

وابسته به جاقى ايجاد كند [f]]. آديبوكاينها و از جمله كمرين، تنظيم كثنده حساسيت به انسولين و ميانجى فرايند التهابي است

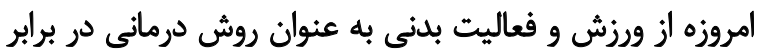

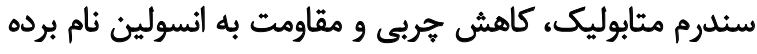

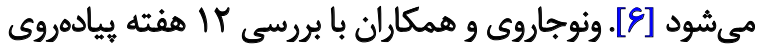

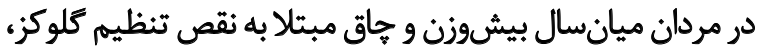

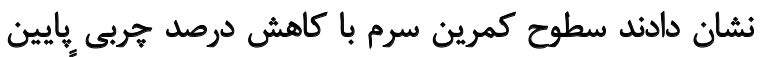

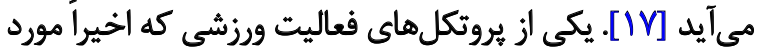

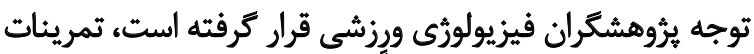

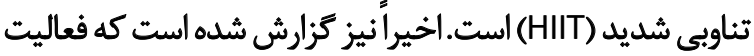

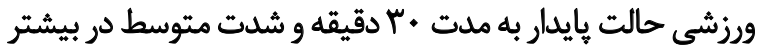

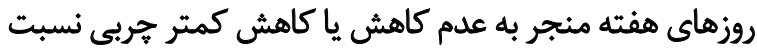

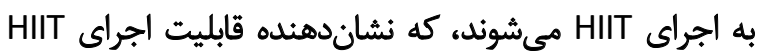

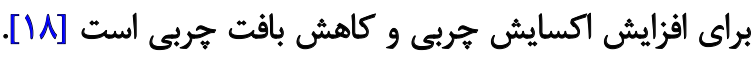

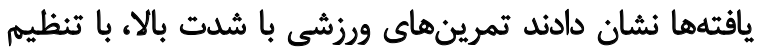

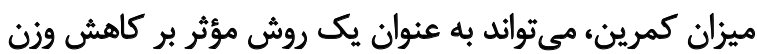

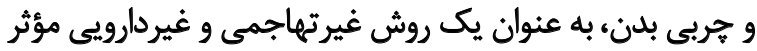

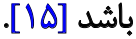
در يروهش حاضر متعاقب هشت هفته تمرين استقامتى مقادير

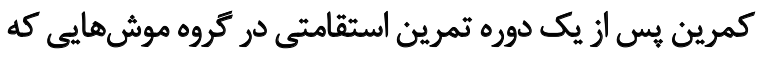

ميزان كمرين در گروهي كه تمرين استقامتى انجام دادهاند نسبت

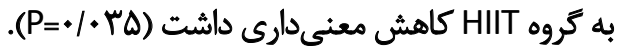

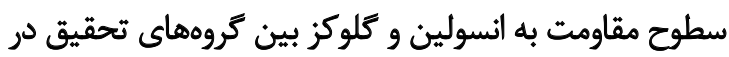

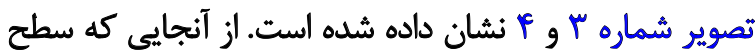

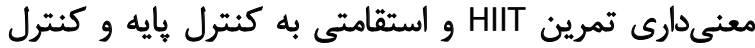

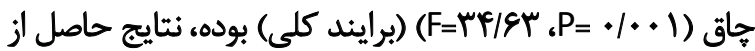

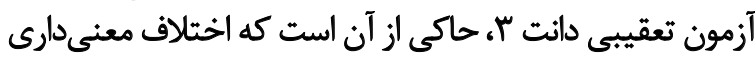
بين سطوح مقاومت به انسولين در كروه تمرينى HIIT نسبت آنسيت

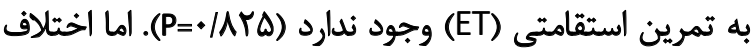

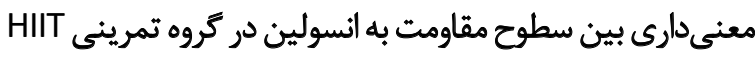

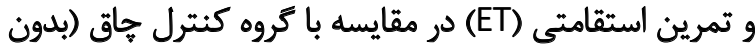
مداخله) و كروه كنترل بايه مشاهده شد.

بحث

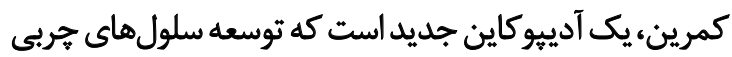

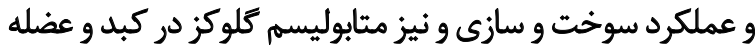

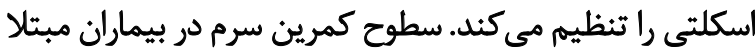

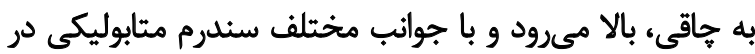

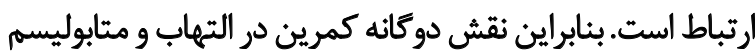

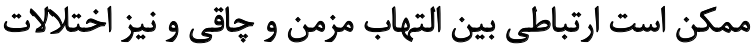

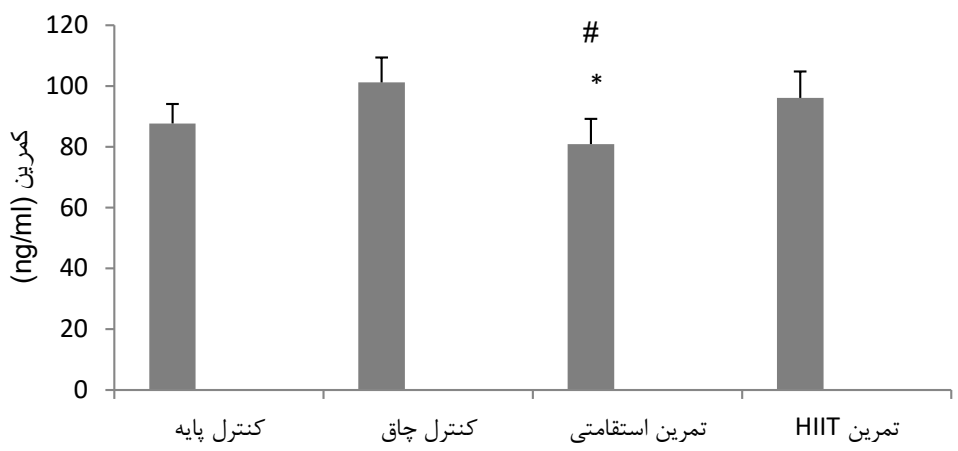

تصوير T. سطوح كمرين يلاسما بين كروههاي تحقيق HIIT تمرين تناوبى شديد

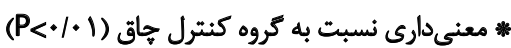

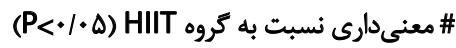




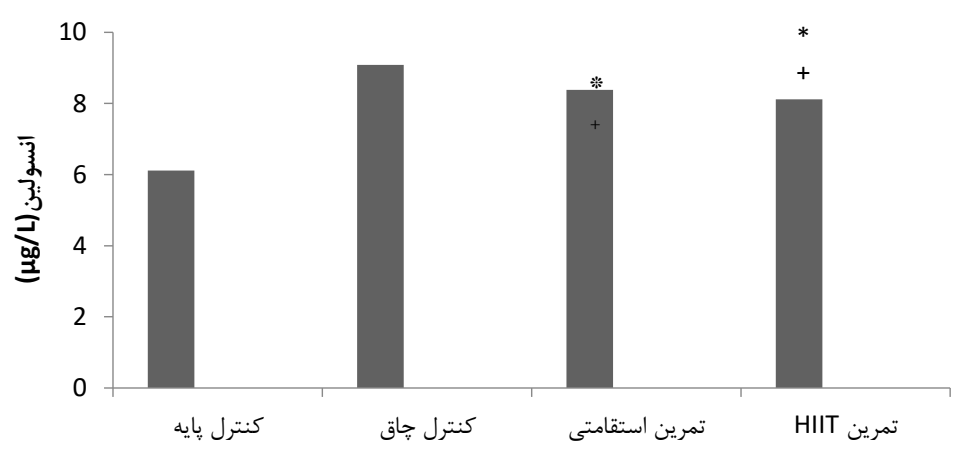

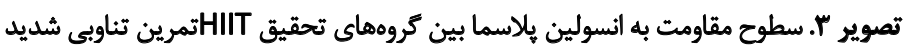

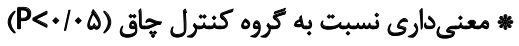

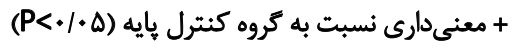

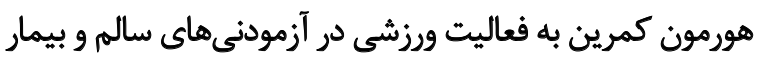

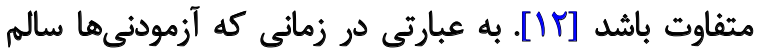

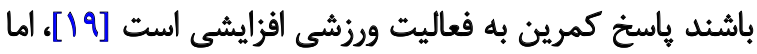

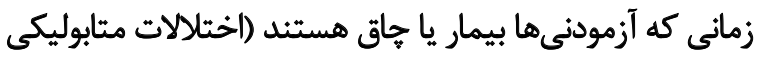

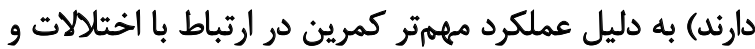

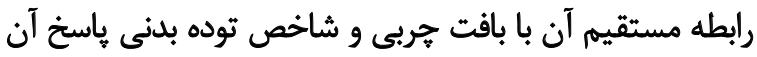

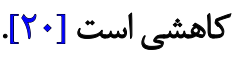

همجنين يافتههاى بُروهش حاضر نشان داد يَّ از يك دوره

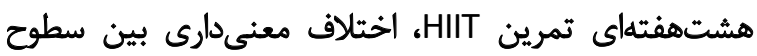

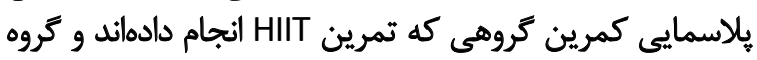

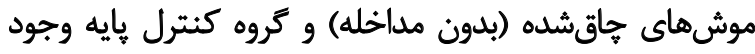

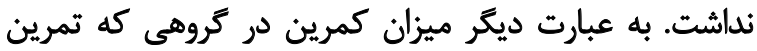

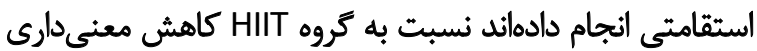

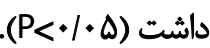

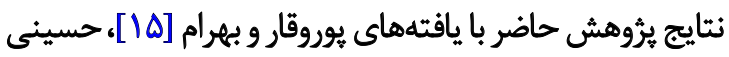

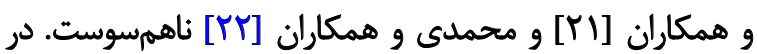

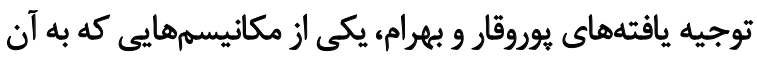

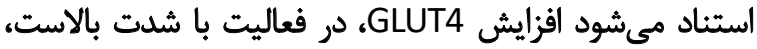

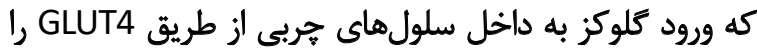

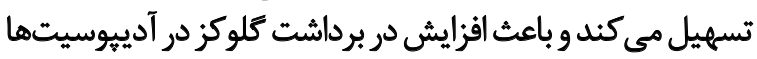

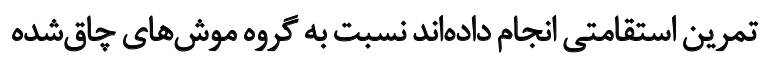

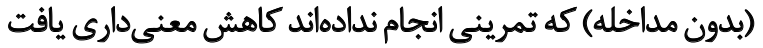

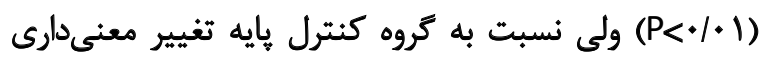

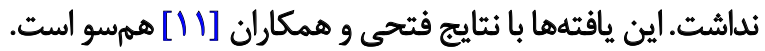

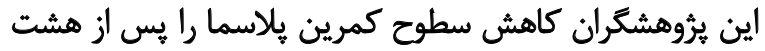

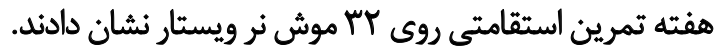

از طرفى نتايج يروهش حاضر با يافتههاى مرادى و همكاران

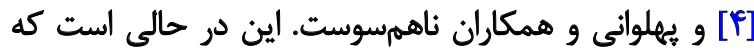

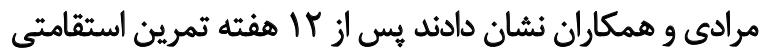

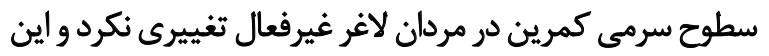

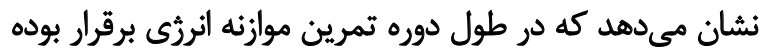

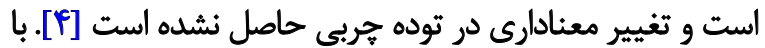

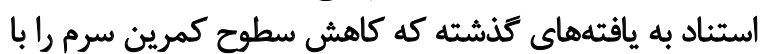

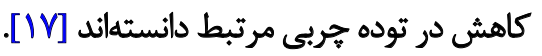
شايد بتوان كفت عدم تغيير سطوح كمرين سرم به دنبال

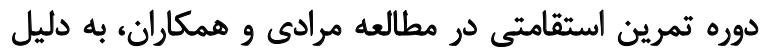

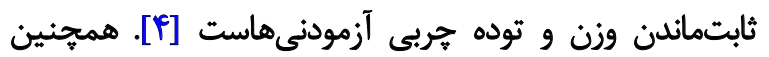

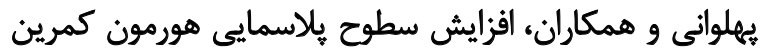

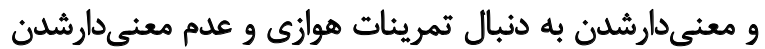

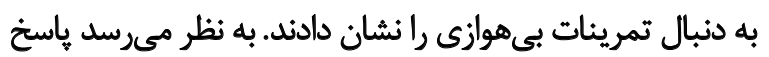

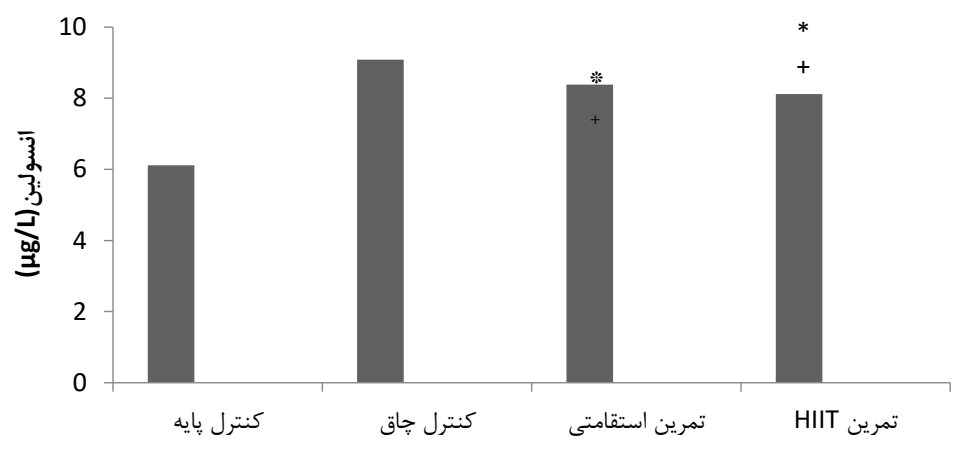


رسيدند كه در سطح انسولين و مقاومت به انسولين به واسطه

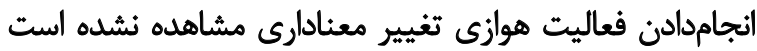

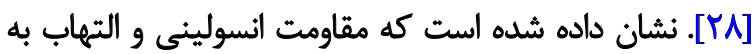

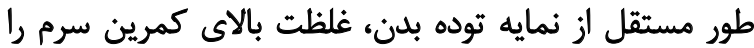

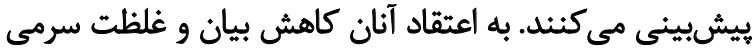

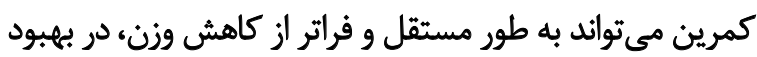
حساسيت انسولينى و التهاب ايفاى نقش كند [ • "r]. ذوالفقارى و همكاران نشان دادند إ هفته تمرين هوازي باعث

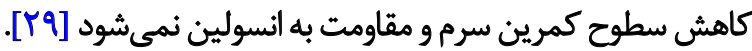

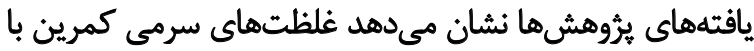

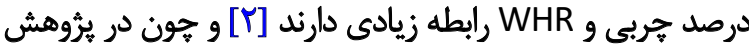

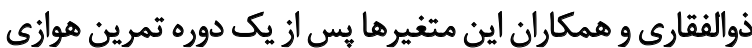

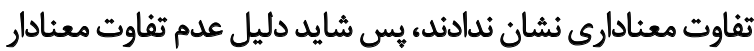

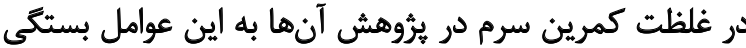

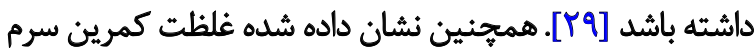

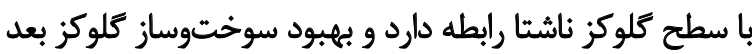

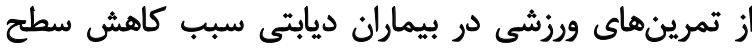

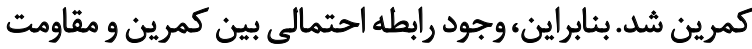

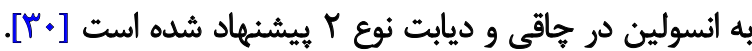

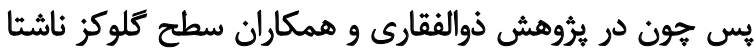

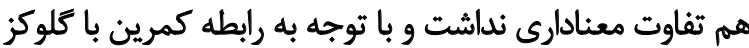

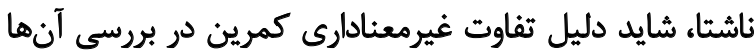
عدم تغيير معنادارى كلوكز باشد.

\section{نتيجِكَّيرى}

با وجود محدوديتها، نتايج يزوهش حاضر نشان داد اجراى

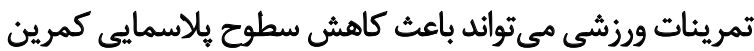

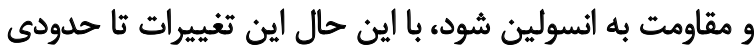

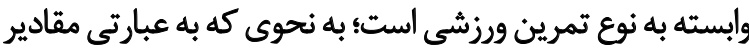

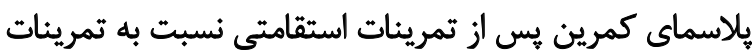

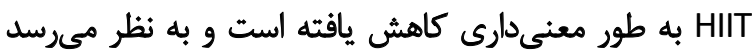

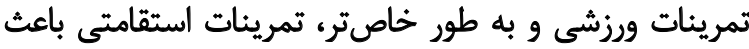
كاهش سطوح كمرين و افزايش حساسيت به انسولين مي شئرد.

مالاحظات اخلاقى

\section{بيوزي أؤ أصول الخالات بُوهش}

اين تحقيق در دانشكده تربيتبدنى و علوم ورزشى دانشكاه

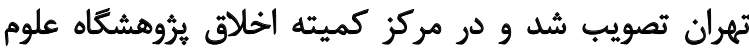
ورزشى با كد IR.SSRI.REC.1398.559 به ثبت وسيت رسيد.

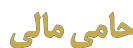

اين تحقيق هيجَّونه حمايت كننده مالى ندارد.
شده و حساسيت به انسولين را در بافت هربي تنظيم مي كني إند

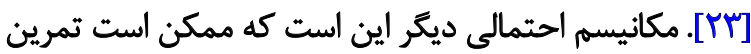

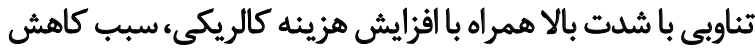

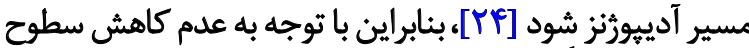

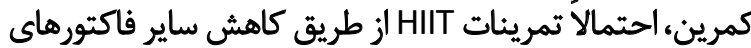

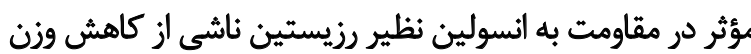

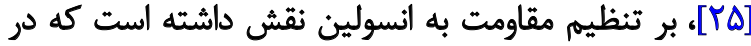
تحقيق حاضر مورد بررسى قرار نكرفته است.

حسينى و همكاران نشان دادند غلظت كمرين و مقاومت

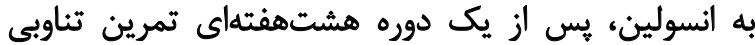

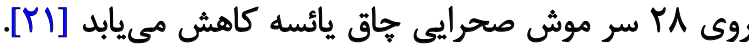

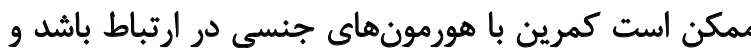

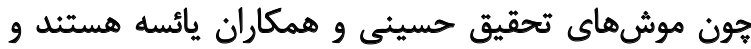

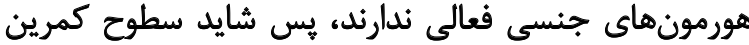

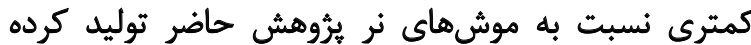

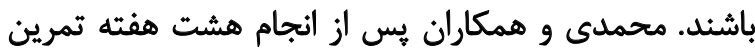
هوازى HIIT

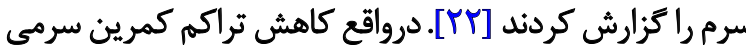

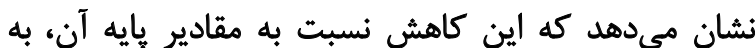

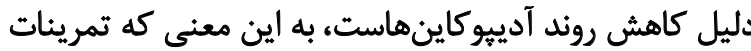

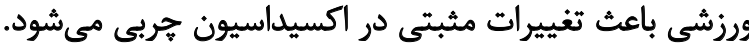

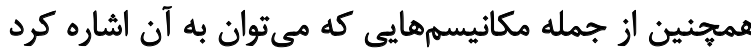

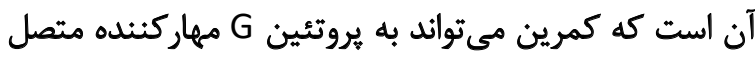

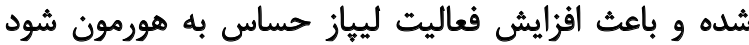

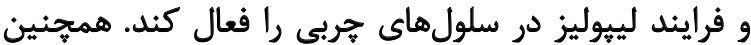

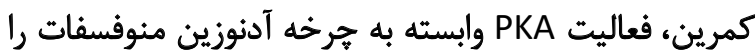

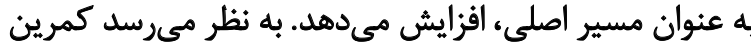

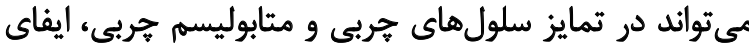

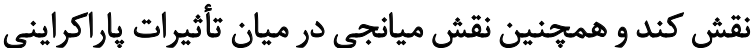

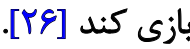

از ديكر نتايج يُوهش حاضر آن بود كه يس از يك دوره

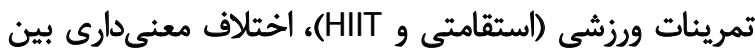

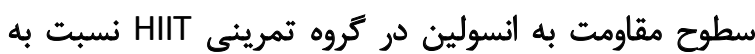

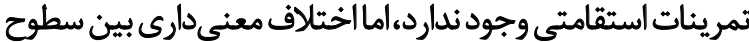

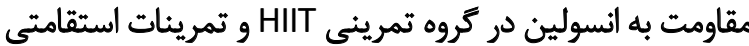

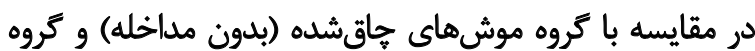

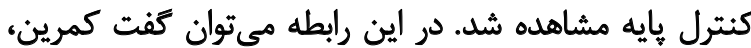

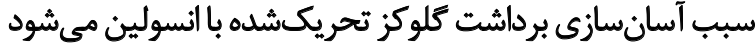

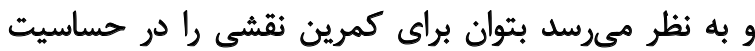

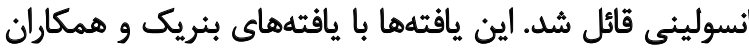

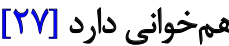

يافتههاى فوق، با يافتههاى هولمي و همكاران [YY] و ذوالفقارى

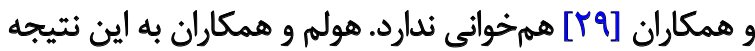




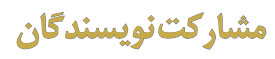

$$
\begin{aligned}
& \text { تمامى نويسندكان به يك اندازه در نكارش مقاله سهيم بودهاند. }
\end{aligned}
$$

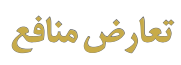

$$
\begin{aligned}
& \text { نويسندكان تصريح مي كنئد هيج كونه تضاد منافعى در خصوص } \\
& \text { بروهش حاضر وجود ندارد. }
\end{aligned}
$$




\section{References}

[1] Kennedy GC. The role of depot fat in the hypothalamic control of food intake in the rat. Proc R Soc Lond B. 1953; 140(901):578-92. [DOI:10.1098/ rspb.1953.0009] [PMID]

[2] Ernst MC, Sinal CJ. Chemerin: At the crossroads of inflammation and obesity. Trends EndocrinolMetabol. 2010; 21(11):660-7. [DOI:10.1016/j. tem.2010.08.001] [PMID]

[3] Saremi A, Moslehabadi M, Parastesh M. [Effects of twelve-week strength training on serum chemerin, tnf- $\alpha$ and crp level in subjects with the metabolic syndrome(Persian)]. IJEM. 2011; 12(5):536-43

[4] Moradi F, Heydarzadeh A, Baneh V. The effect of an endurance training program on serum levels of leptin and chemerin adipokines in inactive lean men. FEYZ. 2014; 18(5):419-27.

[5] Rourke J, Dranse H, Sinal C. Towards an integrative approach to understanding the role of chemerin in human health and disease. Obesity Reviews. 2013; 14(3):245-62. [DOI:10.1111/obr.12009] [PMID]

[6] Sadeghipour H, Daryanoosh F, Salesi M. The effect of 12 weeks of aerobic interval training on chemerin and vaspin serum concentrations and insulin resistance index in overweight students. J Zanjan Univ Med Sci. 2015; 23(96):78-88.

[7] Zhang R, Liu S, Guo B, Chang L, Li Y. Chemerin induces insulin resistance in rat cardiomyocytes in part through the ERK1/2 signaling pathway. Pharmacology. 2014; 94(5-6):259-64. [DOI:10.1159/000369171] [PMID]

[8] Tokmakidis SP, Zois CE, Volaklis KA, Kotsa K, Touvra AM. The effects of a combined strength and aerobic exercise program on glucose control and insulin action in women with type 2 diabetes. Eur J applied physiol. 2004; 92(4-5):437-42. [DOI:10.1007/s00421-004-1174-6] [PMID]

[9] Khalafi M, Shabkhiz F, Aazali Ak, Bakhtiyari A. [Irisin response to two types of exercise training in type 2 diabetic male rats (Persian)]. Aram Med univ J. 2016; 19(111):37-45.

[10] Peeri M, Akbari A, Matin Homaee H. [The effects of aerobic exercise on plasma levels of adiponectin and insulin resistance index in males with Down's Syndrome: A pilot study (Persian)]. Qom Univ Med Sci J. 2015; 9(1-2):14-21.

[11] Fathi M, Delpasand A, Nastaran M. Serum chemerin and insulin sensitivity alterations due to exercise training below and above lactate thresholds in streptozocin-induced diabetic rats. Sport Sci Health. 2015; 11(2):211-5. [DOI:10.1007/s11332-015-0227-z]

[12] Palavani HA, Daryanoosh F, Mohammadi M. The effect of aerobic and anaerobic exercises on changes of chemerin levels in female sprague dawley rats. J Shahid Sadoughi Univ Med Sci. 2014; 22(2):1020-7.

[13] Aghapour A, Farzanegi P. Effect of six-week aerobic exercise on Chemerin and Resistin concentration in hypertensive postmenopausal women. Electronic Physician. 2013; 5(1):623-30.

[14] Gibala MJ, Little JP, MacDonald MJ, Hawley JA. Physiological adaptations to low-volume, high-intensity interval training in health and disease. J Physiol. 2012; 590(5):1077-84. [DOI:10.1113/jphysiol.2011.224725] [PMID] [PMCID]

[15] Pourvaghar $M$, bahram M. [The effect of a three-month intensive intermittent training on plasma chemerin and factors related to body composition on overweight males (Persian)]. 2015; 20(5):381-92.

[16] Sherafati Moghadam M, Daryanoosh F, Mohammadi M, Kooshki Jm, Alizadeh Ph. The effect of eight-week intense sprint exercise on plasma levels of vaspin and chemerin in female sprague-dawley rats. Daneshvar Medicine. 2013; 21(107):0-0.
[17] Venojärvi M, Wasenius N, Manderoos S, Heinonen OJ, Hernelahti M Lindholm $\mathrm{H}$, et al. Nordic walking decreased circulating chemerin and leptin concentrations in middle-aged men with impaired glucose regulation. Ann Med. 2013; 45(2):162-70. [DOI:10.3109/07853890.2012.72 7020] [PMID]

[18] Boutcher SH. High-intensity intermittent exercise and fat loss. J Obes. 2010; 2011:1-10. [DOI:10.1155/2011/868305] [PMID] [PMCID]

[19] Wittamer V, Franssen JD, Vulcano M, Mirjolet JF, Le Poul E, Migeotte I, et al. Specific recruitment of antigen-presenting cells by chemerin, a novel processed ligand from human inflammatory fluids. J Experiment Med. 2003; 198(7):977-85. [DOI:10.1084/jem.20030382] [PMID] [PMCID]

[20] Weigert J, Neumeier M, Wanninger J, Filarsky M, Bauer S, Wiest R et al. Systemic chemerin is related to inflammation rather than obesity in type 2 diabetes. Clinical endocrinology. 2010; 72(3):342-8. [DOI:10.1111/j.1365-2265.2009.03664.x] [PMID]

[21] Hosseini M, Eftekhar B, Riyahi Malayeri S. Effect of interval training with curcumin consumption on some adipokines in menopausal obese rats. J Rafsanjan Univ Med Sci. 2017; 16(6):505-16.

[22] Mohammadi R, Fatdhei M, Ilkhani B. The effect of eight weeks high-intensity interval aerobic training on chemerin and visfatin in overweight men. Beden Egitimi Ve Spor Bilimleri Dergisi. 2017; 11(3):200-6.

[23] Holten MK, Zacho M, Gaster M, Juel C, Wojtaszewski JF, Dela F. Strength training increases insulin-mediated glucose uptake, GLUT4 content, and insulin signaling in skeletal muscle in patients with type 2 diabetes. Diabetes. 2004; 53(2):294-305. [DOI:10.2337/diabetes.53.2.294] [PMID]

[24] Bruun JM, Helge JW, Richelsen B, Stallknecht B. Diet and exercise re duce low-grade inflammation and macrophage infiltration in adipose tissue but not in skeletal muscle in severely obese subjects. Am J Physio Endocrinol and Metabol. 2006; 290(5):E961-E7. [DOI:10.1152/ajpendo.00506.2005] [PMID]

[25] Soori R, Khosravi N, Yazdandost H, Ayati M. A comparison of moderate intensity continuous training and high intensity interval training on serum levels of resistin and insulin resistance in type- 2 diabetic obese women. Sports J Sport Sci. 2016; 8(3):365-80.

[26] Stejskal D, Karpisek M, Hanulova Z, Svestak M. Chemerin is an independent marker of the metabolic syndrome in a caucasian populationa pilot study. Biomedical Paper. 2008; 152(2):217-21. [DOI:10.5507/ bp.2008.033] [PMID]

[27] Benrick A, Maliqueo M, Miao S, Villanueva JA, Feng Y, Ohlsson C, et al. Resveratrol is not as effective as physical exercise for improving reproductive and metabolic functions in rats with dihydrotestosteroneinduced polycystic ovary syndrome. Evi Complement Alter Med. 2013, 2013:964070. [DOI:10.1155/2013/964070] [PMID] [PMCID]

[28] Mannerås Holm L. Polycystic ovary syndrome-studies of metabolic and ovarian disturbances and effects of physical exercise and electroacupuncture [PhD dissertation]. Gothenburg: University of Gothenburg;2010.

[29] Zolfaghari M, Taghian F, Hedayati M. The effects of green tea extract consumption, aerobic exercise and a combination of these on chemerin levels and insulin resistance in obese women. Iran J Endocrinol Metabol. 2013; 15(3):253-61.

[30] Chakaroun R, Raschpichler M, Klöting N, Oberbach A, Flehmig G, Kern $M$, et al. Effects of weight loss and exercise on chemerin serum concentrations and adipose tissue expression in human obesity. Metabolism. 2012; 61(5):706-14. [DOI:10.1016/j.metabol.2011.10.008] [PMID] 
